\title{
Manifestation of structural Higgs and Goldstone modes in the hexagonal manganites
}

\author{
Quintin N. Meier $\odot,{ }^{1, *}$ Adrien Stucky $\odot,{ }^{2}$ Jeremie Teyssier, ${ }^{2}$ Sinéad M. Griffin, ${ }^{3,4}$ \\ Dirk van der Marel, ${ }^{2}$ and Nicola A. Spaldin $\odot^{1}$ \\ ${ }^{1}$ Materials Theory, ETH Zurich, Wolfgang-Pauli-Strasse 27, 8093 Zürich, Switzerland \\ ${ }^{2}$ Department of Quantum Matter Physics, University of Geneva, 24 Quai Ernest-Ansermet, 1211 Geneva 4, Switzerland \\ ${ }^{3}$ Materials Science Division, Lawrence Berkeley National Laboratory, Berkeley, California 94720, USA \\ ${ }^{4}$ Molecular Foundry, Lawrence Berkeley National Laboratory, Berkeley, California 94720, USA
}

(Received 31 January 2020; revised 9 June 2020; accepted 17 June 2020; published 10 July 2020)

\begin{abstract}
Structural phase transitions described by Mexican hat potentials should in principle exhibit aspects of Higgs and Goldstone physics. Here, we investigate the relationship between the phonons that soften at such structural phase transitions and the Higgs- and Goldstone-boson analogs associated with the crystallographic Mexican hat potential. We show that, with the exception of systems containing only one atom type, the usual Higgs and Goldstone modes are represented by a combination of several phonon modes, with the lowestenergy phonons of the relevant symmetry having substantial contribution. Taking the hexagonal manganites as a model system, we identify these modes using Landau theory, and predict the temperature dependence of their frequencies using parameters obtained from density functional theory. Separately, we calculate the additional temperature dependence of all phonon mode frequencies arising from thermal expansion within the quasiharmonic approximation. We predict that Higgs-mode softening will dominate the low-frequency vibrational spectrum of $\mathrm{InMnO}_{3}$ between zero Kelvin and room temperature, whereas the behavior of $\mathrm{ErMnO}_{3}$ will be dominated by lattice expansion effects. We present temperature-dependent Raman scattering data that support our predictions, in particular confirming the existence of the Higgs mode in $\mathrm{InMnO}_{3}$.
\end{abstract}

DOI: 10.1103/PhysRevB.102.014102

\section{INTRODUCTION}

Phase transitions that break a symmetry spontaneously occur in a wide range of physical systems, from low-energy cold atoms, through magnetic, structural, and superconducting transitions in condensed matter, to high-energy collisions at the large hadron collider [1-8]. Perhaps the simplest and most-studied form of spontaneous symmetry breaking is that described by the " $\phi$ " Lagrangian, which is used in the Landau-Ginzburg theory of phase transitions, as well as in the standard model of particle physics,

$$
\mathcal{L}=\frac{1}{2}\left(\partial_{\mu} \phi\right)^{2}-\frac{1}{2} m^{2} \phi^{2}-\frac{\lambda}{4 !} \phi^{4}
$$

where $\phi$ is a complex order parameter which is zero in the disordered phase at $T>T_{C}$ and acquires a nonzero value below $T=T_{C}$. The energy density of such a Lagrangian has the so-called "Mexican hat" potential with continuous U(1) symmetry (see Fig. 1) in which the ground-state value of the field $\phi_{0}$ is degenerate in energy around the entire $360^{\circ}$ rim of the Mexican hat potential. This form was originally

\footnotetext{
*quintin.meier@mat.ethz.ch
}

Published by the American Physical Society under the terms of the Creative Commons Attribution 4.0 International license. Further distribution of this work must maintain attribution to the author(s) and the published article's title, journal citation, and DOI. suggested by Landau to describe ferromagnets near the critical point [9-11] and has recently achieved notoriety following the discovery of the Higgs boson, whose formation it also describes.

Perturbing the field $\phi$ around the ground state $\phi_{0}$ gives two types of fluctuations, the Higgs and Goldstone modes, which correspond respectively to oscillations of the amplitude (toward and away from the peak of the hat) and phase (around the brim of the hat) of the broken continuous symmetry (Fig. 1).

Since the energy of the field is invariant with phase, the Goldstone mode is characteristically massless with zero frequency and a corresponding zero-energy gap [12,13]. Many manifestations of the Goldstone mode are known in condensed-matter systems: For example, a massless spin wave has been measured using neutron scattering in the prototypical Heisenberg ferromagnet EuS [14], for which the Hamiltonian is invariant under the rotation of spins. Inelastic neutron scattering was also used to detect a gapless mode in a Bose-Einstein condensate of spin-triplet states in $\mathrm{TlCuCl}_{3}$ [15], consistent with theoretical predictions [16]. Polarized Raman scattering detected the development of a peak at zero frequency with divergent intensity at the structural phase transition in $\mathrm{Cd}_{2} \mathrm{Re}_{2} \mathrm{O}_{7}$ pyrochlore, which has been associated with the Goldstone phonon [17]; similar behavior has been predicted for Ruddeldsen-Popper-structure $\mathrm{PbSr}_{2} \mathrm{Ti}_{2} \mathrm{O}_{7}$ [18] and Jahn-Teller coupled $d-d$ orbital excitations in the orthorhombic perovskite $\mathrm{LaMnO}_{3}$ [19]. Finally, Goldstone 


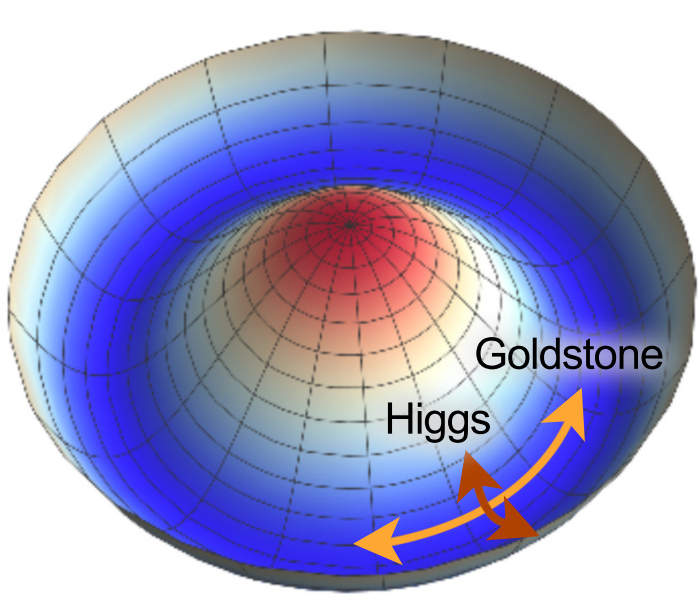

FIG. 1. The Mexican hat potential describing a broken U(1) symmetry, with the Higgs and Goldstone modes indicated.

modes also appear as sound waves at the normal-to-superfluid transition in ${ }^{4} \mathrm{He}$ [20]. In superconductors, the Goldstone mode gains mass by its interaction with an applied external field through the Anderson-Higgs mechanism [21,22], giving rise to the Meissner effect.

In contrast, the Higgs, or amplitude, mode is massive and harmonic [6], with a finite-energy excitation gap. It is harder to detect because it can decay into Goldstone bosons [23], although successful observations have been made in condensed-matter systems for which the effective theory describing the system has a relativistic form. This is the case for the superconducting phase transition in $2 \mathrm{H}-\mathrm{NbSe}_{2}$ [24], which provided the first experimental evidence of the Higgs mode in a condensed-matter system [25] through its unusual Raman response, which was consistent with the occurrence of an amplitude mode of the charge density wave (CDW) order parameter. Likewise, in $\mathrm{TlCuCl}_{3}$, neutron spectroscopy measurements of the magnetic excitations revealed a Higgs mode that softened and vanished at the pressure-induced quantum phase transition from a sea of spin-singlet pairs to a longrange antiferromagnet [26]. Cold atoms in two-dimensional optical lattices have provided indirect measurement of the Higgs mode at the quantum phase transition between the superfluid and insulating phases, through observation of a finite-frequency response in the superfluid phase [27], consistent with Monte Carlo simulations [28] and the scaling expected for a Higgs mode. Recently, the presence of structural Goldstone and Higgs modes was suggested by first-principles calculations on a strained perovskite oxide, $\mathrm{SrMnO}_{3}$ [29]. Finally, the observation and manipulation of a Higgs mode has recently been demonstrated in a supersolid quantum gas [30]. A summary of experimental efforts to observe the Higgs mode in condensed-matter systems can be found in Ref. [31].

Notably, no occurrences of the Higgs mode corresponding to structural phase transitions have been experimentally reported to date. Such an example would be convenient since the order parameters in structural phase transitions are usually given by the positions of the atoms, which in turn can often be measured unambiguously and remain stable for long times. Indeed, a field-theoretical treatment of both Higgs and Goldstone phonons has recently been developed and would in principle be applicable to such a transition [32].

Here, we show that for compounds containing multiple atomic species, an unambiguous association of specific single phonons with the Higgs and Goldstone modes can not in general be made because the different atomic masses of the species cause the eigenvectors of the force constant and dynamical matrices to differ. Nevertheless, we show that phonon modes carrying substantial Higgs and Goldstone character can be identified, and demonstrate their existence in the multiferroic hexagonal manganite family of improper ferroelectrics, which are unusual in that they have a structural phase transition whose energy landscape is described by a Mexicanhat-like potential $[33,34]$. By combining symmetry analysis, first-principles calculations, and phenomenological modeling, we analyze the potential and dynamical energy landscapes of two representative hexagonal manganite materials $\mathrm{ErMnO}_{3}$ and $\mathrm{InMnO}_{3}$. We evaluate the signatures of Higgs-Goldstone coupling in the temperature dependence of the phonon frequencies, and separate these from frequency shifts due to thermal lattice expansion. We then use temperature-dependent Raman spectroscopy to verify the predicted behavior. We find that, while the behavior of $\mathrm{ErMnO}_{3}$ is dominated by lattice expansion effects up to room temperature, Higgs-mode softening can be clearly identified in the vibrational spectrum of $\mathrm{InMnO}_{3}$, providing a crystallographic Higgs mode analog associated with the structural phase transition.

\section{A. Structural Higgs and Goldstone modes in multispecies crystalline materials}

We begin by reviewing the approximations inherent in reducing the large number of structural modes associated with the many atomic displacement degrees of freedom in a solid to the effective theory $\phi^{4}$ theory of Eq. (1) in terms of the Higgs and Goldstone modes. Expanding the total energy of a system of atoms around their ground-state positions in the zero-temperature structure, one obtains the total energy of the system $E$ as the sum of its kinetic and potential energies:

$$
\begin{aligned}
E= & \frac{1}{2} \sum_{i} m_{i}\left(\partial_{t} u_{i}\right)^{2}+\sum_{i, j} \Phi_{i j}(0) u_{i} u_{j}+\sum_{i j k} \Lambda_{i j k} u_{i} u_{j} u_{k} \\
& +\frac{1}{4} \sum_{i, j, k, l} \Pi_{i j k l} u_{i} u_{j} u_{k} u_{l}-\cdots
\end{aligned}
$$

where $i \in\{1 x, 1 y, 1 z, 2 x, 2 y, 2 z, \ldots, N x, N y, N z\}$ can be a large number, leading to a large number of modes, even when periodic boundary conditions are used to constrain $N$ to the number of atoms in the unit cell. Here, $\frac{1}{2} \sum_{i} m_{i}\left(\partial_{t} u_{i}\right)^{2}$ is the kinetic energy of the atoms, $\Phi_{i j}(0)=\frac{\partial^{2} E}{\partial u_{i} \partial u_{j}}$ is the harmonic force constant matrix at zero temperature $(E$ is the internal energy and $u_{i}, u_{j}$ are displacements of the $i$ th and $j$ th atoms from their positions in the zero-temperature structure), and $\Lambda_{i j k}$ and $\Pi_{i j k l}$ are the anharmonic third- and fourth-order force constant matrices. 
At low temperatures, the amplitudes of the atomic displacements are small, the average atomic positions are unchanged from the zero-temperature positions, and the phonon eigenmodes are obtained by diagonalizing the sum of the first two terms. As temperature is increased, the anharmonic couplings become relevant, leading to two effects: First, the normal modes can no longer be separated into the zerotemperature phonons and the average positions of the atoms are changed, leading to the well-established lattice expansion in conventional solids; we treat this behavior later in Sec. III C. Second, in systems close to a structural phase transition, the response is dominated by an additional change of atomic positions associated with the anharmonicity of a single softmode coordinate; we focus on this behavior here.

To avoid calculation of the full partition function of Eq. (2), the anharmonicities are renormalized into a harmonic approximation around the new atomic positions at each temperature. A renormalized force constant matrix $\Phi_{i j}(T)=\frac{\partial^{2} F(T)}{\partial u_{i}^{*} \partial u_{j}^{*}}$ then describes the energy cost of small atomic displacements $u_{i}^{*}$ away from the minimum-energy atomic coordinates at temperature $T$. In this renormalized harmonic approximation the total free energy $F(T)$ is given by

$$
F(T)=F_{0}(T)+\frac{1}{2} \sum_{i} m_{i}\left(\partial_{t} u_{i}^{*}\right)^{2}+\sum_{i j} \Phi_{i j}(T) u_{i}^{*} u_{j}^{*}
$$

where $F_{0}(T)$ is the free energy of the minimum energy structure $\left(u_{i}^{*}=0\right)$ at the temperature $T$.

Next, we use the Landau theory of phase transitions to analyze the finite-temperature force-constant matrix, assuming that the temperature evolution is fully captured by the evolution of the two eigenvectors ( $\phi_{1}$ and $\phi_{2}$ say) that represent the two-dimensional order parameter and form the soft mode. We can then write the free energy in the usual Landau form for a broken continuous U(1) symmetry [35] in terms of the two-component order parameter $\phi=\left(\phi_{1}, \phi_{2}\right)$ as

$$
F=\frac{a(T)}{2}\left(\phi_{1}^{2}+\phi_{2}^{2}\right)+\frac{b}{4}\left(\phi_{1}^{2}+\phi_{2}^{2}\right)^{2}+\cdots
$$

which has the same form as Eq. (1). Here,

$$
a(T)= \begin{cases}>0 & \text { if } T>T_{C} \\ 0 & \text { if } T=T_{C} \\ <0 & \text { if } T<T_{C}\end{cases}
$$

This approximation implies that the temperature dependence of the energy landscape is determined entirely by the anharmonicity in these two soft modes with no anharmonic coupling to other modes, and at any temperature, $\Phi_{i j}(T)$ is diagonalized by the same basis set, with the anharmonicities confined in the subspace $\left(\phi_{1}, \phi_{2}\right)$.

Next we calculate the eigenvalues $\frac{\partial^{2} F}{\partial \phi_{i}^{2}}$, of these two modes, above and below $T_{C}$. Above $T_{C}$, the expectation values of $\phi_{1}$ and $\phi_{2}$ are equivalently zero, and so

$$
\left.\frac{\partial^{2} F}{\partial \phi_{1}^{2}}\right|_{\phi_{1}=0, \phi_{2}=0}=\left.\frac{\partial^{2} F}{\partial \phi_{2}^{2}}\right|_{\phi_{1}=0, \phi_{2}=0}=a(T) .
$$

Below $T_{C}$, the two modes correspond to the Goldstone and Higgs modes. The Goldstone mode has an eigenvalue of zero for all temperatures below the phase transition. The Higgs mode, in contrast, softens with increasing temperature, so that its eigenvalue goes to zero at the phase transition. Formally, we obtain the solutions below $T_{C}$ by minimizing the free energy $F$ [Eq. (4)] with respect to $\phi_{1}$ and $\phi_{2}$ to extract the expectation value of the order parameter. This yields two solutions, the trivial vacuum solution with $\left\langle\phi_{1}\right\rangle=\left\langle\phi_{2}\right\rangle=0$ for $-a(T) / b>0$, and a nontrivial solution describing a degenerate circle of vacua $\phi_{1}^{2}+\phi_{2}^{2}=\langle\phi\rangle^{2}=-a(T) / b$, which is the Mexican hat potential. Because of the U(1) symmetry we can choose $\left\langle\phi_{1}\right\rangle=0$ and $\left\langle\phi_{2}\right\rangle=\langle\phi\rangle$ without loss of generality, and expand around the low-symmetry vacuum ground state to obtain the excitation modes. This gives the massless Goldstone mode, corresponding to distortions along the $\phi_{1}$ coordinate around the brim of the hat, and the massive Higgs mode, corresponding to distortions along the perpendicular $\phi_{2}$ coordinate.

The frequencies of the Goldstone and Higgs modes are

$$
\begin{gathered}
\left.\frac{\partial^{2} F}{\partial \phi_{1}^{2}}\right|_{\phi_{1}=0, \phi_{2}=\langle\phi\rangle}=0, \\
\left.\frac{\partial^{2} F}{\partial \phi_{2}^{2}}\right|_{\phi_{1}=0, \phi_{2}=\langle\phi\rangle}=a(T)+3 b\langle\phi\rangle^{2}=-2 a(T)
\end{gathered}
$$

with the eigenvalues of all other modes still temperature independent. We assume therefore a strong temperature dependence of the eigenvalue of the force-constant matrix corresponding to the Higgs mode on approaching the structural phase transition, with the remaining eigenvalues being largely temperature independent.

Finally, for this section we emphasize that, since the Mexican hat is a potential energy surface, the Higgs and Goldstone modes are the relevant eigenmodes of the force-constant matrix. There is no obvious way, however, to directly measure the eigenmodes of the force-constant matrix and, therefore, the phonon modes, which are readily accessible via vibrational spectroscopies, are often used as proxies. The phonon modes, however, are eigenmodes of the dynamical matrix, which is related to the force-constant matrix by (see, for example, Ref. [36]):

$$
D_{i j}=\frac{\Phi_{i j}}{\sqrt{M_{i} M_{j}}},
$$

where $M_{i}$ is the mass of the $i$ th atom. It is clear that the forceconstant and dynamical matrices have different eigenvalues and eigenvectors. As a result, the Higgs and Goldstone modes associated with a crystallographic phase transition do not correspond to single phonons, except in the special case that the system contains atoms of only one mass $M$, in which case the eigenvectors of the force constants and dynamical matrices are the same, and the phonon frequencies corresponding to the Goldstone and Higgs modes $\omega_{G}$ and $\omega_{H}$ are given by

$$
\begin{aligned}
& \omega_{G}=\sqrt{\alpha_{1} / M}, \\
& \omega_{H}=\sqrt{\alpha_{2} / M},
\end{aligned}
$$


(a)

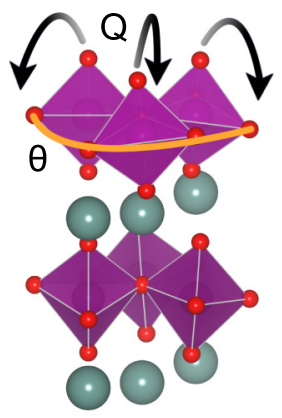

(b)

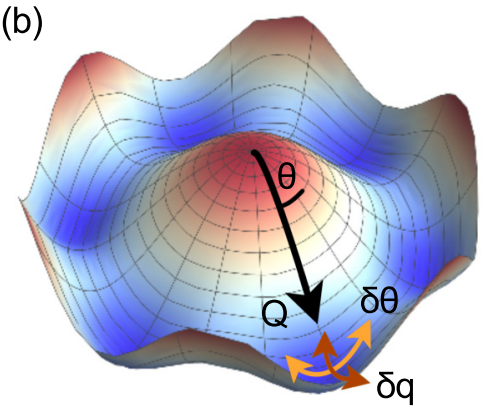

FIG. 2. (a) Crystal structure of the hexagonal manganites, black arrows indicating the tilt amplitude $Q$ and orange circle indicating the tilt angle $\theta$ of the oxygen-manganese bipyramids. The oxygens are marked red, the manganese atoms are purple, and the $R$ atoms are green. (b) Mexican hat free-energy landscape showing the energy as a function of amplitude $(Q)$ and phase $(\theta)$ of the order parameter. The U(1)-like symmetry at small order parameter values, and the six discrete minima at large order parameter values can be clearly seen. The fluctuations in the amplitude $\delta q$ (Higgs) and in the phase $\delta \theta$ (Goldstone) are indicated with red and orange arrows.

where $\alpha_{1}$ and $\alpha_{2}$ are the corresponding eigenvalues of the force-constant matrix. In a general multicomponent system, however, each element of the force-constant matrix must be divided by the product of the square roots of the relevant masses before diagonalization to extract the phonons, and in general the static eigenvectors of the force-constant matrix do not correspond to specific single phonons. For the special case of the zero-frequency Goldstone mode, the atomic masses are not relevant, and as a result there is a zero-frequency phonon for each zero-frequency force-constant mode, and the zero-frequency eigenvectors of the force-constant matrix are identical to the atomic displacements of the corresponding zero-frequency phonon mode. The static Higgs mode, however, is a linear combination of all dynamical phonon modes with the same irreducible representation, and the entire subspace of phonon modes with the same symmetry as the Higgs mode should exhibit the strong temperature dependence that we derived above. A detailed description of the generation of the force-constant matrices and the calculation of the frequencies can be found in the Appendix.

\section{B. Structural phase transition in the hexagonal manganites}

The multiferroic hexagonal manganites consist of layers of corner-sharing $\mathrm{MnO}_{5}$ trigonal bipyramids separating hexagonal planes of $R$ ions ( $R=\mathrm{In}, \mathrm{Sc}, \mathrm{Y}$, or Dy-Lu). They undergo a spontaneous symmetry-breaking structural phase transition between a high-temperature centrosymmetric $P 6_{3} / \mathrm{mmc}$ phase and a ferroelectric $P 6_{3} \mathrm{~cm}$ structure. The primary order parameter is defined by trimerizing tilts of the $\mathrm{MnO}_{5}$ trigonal bipyramids, and is two dimensional, with its amplitude set by the magnitude of the tilt, and its phase set by the tilt angle. The crystal structure and the distortion are illustrated in Fig. 2(a). A combination of Landau theory and first-principles calculations $[33,37,38]$ have shown that

for small tilt amplitudes, the energy is independent of the polyhedral tilt angle, and so near the phase transition the energy landscape can be described by a continuous Mexican hat potential with U(1) symmetry [see Fig. 2(b)]. Thus, unusually for a crystallographic transition, the structural phase transition in the hexagonal manganites is described by a continuous primary two-dimensional order parameter $\left(\phi_{1}, \phi_{2}\right)$ with an energy landscape similar to the one analyzed in the previous section. As such, it might be expected to display Higgs and Goldstone modes. Importantly, the chemistry of the $R$ ion can strongly modify the details of the energy landscape, causing differences in the height of the peak in the Mexican hat potential and in turn influencing the transition temperature; for the $\mathrm{ErMnO}_{3}$ and $\mathrm{InMnO}_{3}$ considered in this work the transition temperatures are 1200 and $500 \mathrm{~K}$, respectively [39].

We note that the discreteness of the lattice manifests at larger amplitudes of the tilt mode through coupling to a secondary ferroelectric order parameter $P$, corresponding to a net shift of the rare-earth ions relative to the manganese oxygen layers along the vertical axis [37]. This mode has shown to be irrelevant in the region of the phase transition [40], a concept referred to as dangerous irrelevance [41]. The recent demonstration that the hexagonal manganites disorder continuously on all length scales close to $T_{c}$ [42] reinforces the continuous $\mathrm{U}(1)$ behavior in the region of the phase transition.

The coupling between $P$ and the primary order parameter yields a low-temperature ground state with six minima around the brim of the hat reflecting the hexagonal symmetry so that the transition deviates from the ideal field theory of Eq. (1) and is instead described by an extended Landau free energy, which is conventionally written in the form [33]

$$
\begin{aligned}
F= & \frac{a}{2} Q^{2}+\frac{b}{4} Q^{4}+\frac{1}{6}\left(c+c^{\prime} \cos 6 \theta\right) Q^{6} \\
& -g Q^{3} P \cos 3 \theta+\frac{g^{\prime}}{2} Q^{2} P^{2}+\frac{a_{p}}{2} P^{2} .
\end{aligned}
$$

For consistency with the hexagonal manganites literature, we use polar coordinates for the order parameter, with the amplitude $Q=\sqrt{\phi_{1}^{2}+\phi_{2}^{2}}$ and the phase $\theta=\arctan \left(\phi_{1} / \phi_{2}\right)$. The energy landscape of the primary order parameter corresponds to an almost perfect Mexican hat, while the secondary order parameter induces the minima in the brim [33]. The energy landscape for a minimized secondary order parameter is shown in Fig. 2(b) for the case of $\mathrm{ErMnO}_{3}$. The detailed chemistry affects the coupling to the polar mode, and thus the height of the barriers around the brim of the hat. In particular, in the case of $\mathrm{InMnO}_{3}$ these are close to zero and the brim is smoother than that shown here [43]. Perturbations of the phase $\delta \theta$ and the amplitude $\delta q$ of the order parameter are also indicated. Perturbations of the amplitude conserve the spacegroup symmetry, thus they belong to the irreducible representation A1. Perturbations of the order-parameter angle change the space-group symmetry from the ferroelectric $P 6_{3} \mathrm{~cm}$ to $P 3 c 1$, which corresponds to the irreducible representation B1 [42]. 


\section{METHODS}

\section{A. Sample preparation}

Thin platelets of $\mathrm{ErMnO}_{3}$ were prepared using the $\mathrm{PbO}-\mathrm{PbF}_{2}$ flux method as described in Refs. [44,45]. The $\mathrm{InMnO}_{3}$ samples were prepared as described in Ref. [46]. The energy balance between the usual polar $P 6_{3} \mathrm{~cm}$ and an antipolar $P \overline{3} c 1$ structure, formed at tilt angles halfway between those of the usual polar structures, is known to be sensitive to the details of the defect chemistry in $\mathrm{InMnO}_{3}[43,47,48]$, and samples with the two phases were obtained by appropriate annealing treatment.

\section{B. Raman spectroscopy}

We performed Raman spectroscopy using a home-made spectrometer equipped with a liquid-nitrogen-cooled CCD camera and an Ar laser for the excitation with a wavelength of $514.5 \mathrm{~nm}$. The Raman spectra were collected at the Stokes side of the elastic peak in the range from 50 to $850 \mathrm{~cm}^{-1}$. Samples were mounted in a compact flow cryostat operating between 4 and $300 \mathrm{~K}$. The power of the laser was low enough to limit local heating of the sample. Of the six different irreducible representations that classify the phonon modes in the hexagonal manganites, only A1 and E2 are Raman active. We use two configurations of the polarization of the incoming and scattered photons: For the $z(x x) \bar{z}$ (polarization of the scattered photons is parallel to that of the incoming ones), both A1 and E2 modes are allowed by the Raman selection rules. For the $z(x y) \bar{z}$ configuration (scattered photons are polarized perpendicular to the incoming ones), only E2 modes can be observed. The relative angle of the two polarizers was calibrated using the selection rules for the $514-\mathrm{cm}^{-1}$ phonon line of silicon.

\section{Density functional calculations}

For our first-principles calculations we used density functional theory as implemented in the ABINIT code $[49,50]$. We treated the exchange-correlation functional within the LDA + $U$ approximation, with $U$ and $J$ values of 8 and $0.88 \mathrm{eV}$ [51], and the core electrons using the projector augmented wave (PAW) method [52] from the JTH pseudopotential table provided by the ABINIT PSEUDODOJO [53]. We used a cutoff energy of 30 hartree and $\Gamma$-centered $k$-point meshes of $8 \times 8 \times 2$ for the 10 -atom unit cells, and $6 \times 6 \times 2$ for the 30 -atom unit cells. Note that with these parameters $\mathrm{InMnO}_{3}$ is ferroelectric; small adjustments in the parameters can stabilize the antipolar $P \overline{3} c 1$ state [54]. We obtained force-constant matrices using the finite-displacement method provided in the PHONOPY package [55]. We calculated the Landau parameters for $\mathrm{ErMnO}_{3}$ by displacing the atoms from their positions in the high-symmetry structure along the force-constant eigenvectors. For our calculations within the quasiharmonic approximation, we calculated the phonons in 30-atom unit cells and computed the internal energies and phonon free energies as a function of in-plane and out-of-plane lattice parameters. We then interpolated between the calculated values to extract the minimum energy lattice parameters at each temperature.

\section{THEORETICAL RESULTS}

\section{A. Density functional calculation of zero-Kelvin energetics and lattice dynamics}

We begin by comparing the zero-temperature energetics of our two representative hexagonal manganites, $\mathrm{ErMnO}_{3}$ and $\mathrm{InMnO}_{3}$. As stated above, the different chemistries of the two materials lead to quantitative differences in their Mexican hat potentials, with a hat height of $\sim 500$ (170) $\mathrm{meV}$ and a barrier in the brim of $\sim 200(50) \mathrm{meV}$ for $\mathrm{ErMnO}_{3}\left(\mathrm{InMnO}_{3}\right)$. The calculated Landau parameters from which these values were obtained are given in Table I.

Next, we calculate the phonon mode frequencies and eigenvectors for the two materials using density functional theory. Our calculated frequencies and the symmetries of each mode are listed in Table IV of Appendix B. We see that the lowest-frequency A1 mode, which we expect to have the largest Higgs character, has almost the same frequency $\left(\sim 130 \mathrm{~cm}^{-1}\right)$ in both materials, reflecting the similar curvatures of their Mexican hat potentials in the brim of the hat in the direction toward and away from the peak. The lowestfrequency B1 modes, which we expect to have the strongest Goldstone character, are strikingly different, however, with the frequency in $\mathrm{ErMnO}_{3}\left(\sim 107 \mathrm{~cm}^{-1}\right)$ considerably higher than that in $\mathrm{InMnO}_{3}\left(\sim 65 \mathrm{~cm}^{-1}\right)$. This is consistent with the larger barriers around the brim of the hat in the $\mathrm{ErMnO}_{3}$ case. Note that even in the case of $\mathrm{InMnO}_{3}$, where the brim of the hat is very smooth, the frequency is still quite far from zero.

Finally, in anticipation of differences in the HiggsGoldstone coupling caused by the different Mexican hats, we calculate the phonon-phonon couplings between the lowfrequency A1 and B1 modes in the two materials. Our results are presented in Table II, with the form of the coupling given by

$$
\begin{aligned}
& E_{\text {phonon-phonon }}={\frac{\omega_{\mathrm{A} 1}^{2}}{2}}^{2} A_{\omega_{\mathrm{A} 1}}^{2}+{\frac{\omega_{\mathrm{B} 1}}{2}}^{2} A_{\omega_{\mathrm{B} 1}}^{2}+c A_{\omega_{\mathrm{A} 1}}^{3}+d A_{\omega_{\mathrm{A} 1}} A_{\omega_{\mathrm{B} 1}}^{2} \\
& +e A_{\omega_{\mathrm{A} 1}}^{4}+f A_{\omega_{\mathrm{B} 1}}^{4}+g A_{\omega_{\mathrm{A} 1}}^{2} A_{\omega_{\mathrm{B} 1}}^{2} .
\end{aligned}
$$

For the lowest-lying A1 and B1 modes, we observe that the lowest-order coupling term $d$ is larger in $\mathrm{InMnO}_{3}$ than in $\mathrm{ErMnO}_{3}$.

\section{B. Landau theory calculation of the temperature dependence of the Higgs- and Goldstone-like phonon modes}

Next, we use Landau theory based on our calculated density functional theory parameters to calculate the explicit temperature dependence of the phonon frequencies. We begin by extending the Landau theory framework that we developed in Sec. I A to include, in addition to the two-component primary order parameter, the secondary order parameter that is relevant in the hexagonal manganites. We then calculate explicitly the temperature dependence of the phonons in both $\mathrm{ErMnO}_{3}$ and $\mathrm{InMnO}_{3}$ using the coefficients of Table I. We proceed by expanding the free energy of Eq. (A11) in terms of small perturbations of the primary (treating each component separately) and secondary order parameters around the minimum energy positions $\theta=0+\delta \theta, Q=\tilde{Q}+\delta q, P=$ $\tilde{P}+\delta p$. Here, $\tilde{Q}$ is the expectation value of $Q$, given by the solution of the equation $\left.\frac{\partial F}{\partial Q}\right|_{\theta=0, P=\tilde{P}}=0$. Correspondingly, $\tilde{P}$ is 
TABLE I. Landau parameters for $\mathrm{ErMnO}_{3}$ (calculated in this work) and $\mathrm{InMnO}_{3}$ (from Ref. [56]).

\begin{tabular}{lccccccc}
\hline \hline & $a\left(\mathrm{eV} \AA^{-2}\right)$ & $b\left(\mathrm{eV}^{-4}\right)$ & $c\left(\mathrm{eV} \AA^{-6}\right)$ & $c^{\prime}\left(\mathrm{eV} \AA^{-6}\right)$ & $g\left(\mathrm{eV}^{-4}\right)$ & $g^{\prime}\left(\mathrm{eV}^{-4}\right)$ & $a_{p}\left(\mathrm{eV} \AA^{-2}\right)$ \\
\hline $\mathrm{ErMnO}_{3}$ & -3.83 & 6.20 & 1.06 & 0.06 & 3.76 & 17.43 \\
$\mathrm{InMnO}_{3}$ & -0.82 & 1.13 & 0.81 & 0.04 & 1.02 & 4.85 \\
\hline \hline
\end{tabular}

the expectation value of $P$, which we obtain from the solution of $\left.\frac{\partial F}{\partial P}\right|_{Q=\tilde{Q}, \theta=0}=0$, yielding

$$
\tilde{P}[Q, \theta]=\frac{g Q^{3} \cos 3 \theta}{g^{\prime} Q^{2}+a_{p}} .
$$

We obtain the following effective susceptibilities for the perturbations of each component:

$$
\begin{gathered}
\chi_{\delta q}^{-1}=a(T)+3 b \tilde{Q}^{2}+5 b \tilde{Q}^{4}\left(c+c^{\prime}\right)-6 g \tilde{Q} \tilde{P}+g^{\prime} \tilde{P}^{2}, \\
\chi_{\delta \theta}^{-1}=-6 c^{\prime} \tilde{Q}^{4}+9 g \tilde{Q} \tilde{P} \\
\chi_{\delta p}^{-1}=g^{\prime} \tilde{Q}^{2}+a_{p} .
\end{gathered}
$$

We then calculate the phonon frequencies at each temperature by replacing the calculated zero-temperature susceptibilities by the response functions (A13)-(A15) in the force-constant matrix, and assuming the usual linear evolution $a(T)=a_{0}\left(T-T_{c}\right) / T_{c}$ for the temperature dependence of the $a$ parameter of the soft mode. Our calculated phonon frequen-

TABLE II. Phonon-phonon coupling between A1 and B1 modes in $\mathrm{InMnO}_{3}$ (top) and $\mathrm{ErMnO}_{3}$ (bottom), calculated in this work. Frequencies are given in $\mathrm{cm}^{-1}$, units of the coupling constants are $\mathrm{meV}(\mathrm{amu} \AA)^{-3 / 2}$ for $\mathrm{c}$ and $\mathrm{d}$, and $\mathrm{meV} /(\mathrm{amu} \AA)^{-2}$ for e-g. Slight differences in the frequencies to the calculated values are due to fitting. Only the lowest-lying A1 and B1 modes were considered.

\begin{tabular}{ccccccc}
\hline \hline \multicolumn{7}{c}{$\mathrm{InMnO}_{3}$} \\
\hline$\omega_{A 1}$ & $\omega_{B 1}$ & $\mathrm{c}$ & $\mathrm{d}$ & $\mathrm{e}$ & $\mathrm{f}$ & $\mathrm{g}$ \\
\hline 127.8 & 63.4 & 0.9 & 9.2 & 0.2 & 0.5 & 1.5 \\
127.9 & 204.8 & 0.9 & 1.5 & 0.2 & 0.7 & 0.6 \\
202.9 & 63.4 & 2.8 & 11.0 & 0.7 & 0.5 & 2.7 \\
202.9 & 204.8 & 2.8 & 2.0 & 0.7 & 0.7 & 1.4 \\
236.0 & 63.6 & -6.7 & 2.5 & 1.7 & 0.5 & 0.2
\end{tabular}

$\mathrm{ErMnO}_{3}$

\begin{tabular}{lcccccc}
\hline$\omega_{A 1}$ & $\omega_{B 1}$ & $\mathrm{c}$ & $\mathrm{d}$ & $\mathrm{e}$ & $\mathrm{f}$ & $\mathrm{g}$ \\
\hline 129.6 & 106.1 & 0.8 & 3.3 & 0.0 & 0.1 & 0.1 \\
129.6 & 267.0 & 0.9 & 3.9 & 0.1 & 0.9 & 0.5 \\
129.6 & 273.4 & 0.8 & 5.3 & 0.1 & 0.8 & 0.2 \\
242.3 & 105.9 & 7.8 & 7.4 & 0.8 & 0.1 & 0.9 \\
242.5 & 267.5 & 8.0 & 10.3 & 0.8 & 0.8 & 2.5 \\
242.6 & 274.1 & 7.9 & 3.1 & 0.7 & 0.5 & 1.1 \\
273.6 & 105.4 & -7.7 & 4.6 & 0.4 & 0.2 & 0.3 \\
273.3 & 267.2 & -7.7 & 17.0 & 0.5 & 0.8 & 0.2 \\
273.4 & 273.6 & -7.8 & 19.8 & 0.5 & 0.7 & 0.7 \\
314.5 & 105.6 & -8.9 & -2.2 & 1.3 & 0.2 & 0.0 \\
314.5 & 267.6 & -8.9 & -7.6 & 1.4 & 0.7 & 1.8 \\
314.5 & 274.1 & -8.9 & 11.6 & 1.4 & 0.5 & 1.0 \\
\hline \hline
\end{tabular}

cies as a function of temperature are shown in Fig. 3(a) for $\mathrm{InMnO}_{3}$ and in Fig. 3(b) for ErMnO 3 . Modes of A1 symmetry (and therefore Higgs character) are indicated in blue, B1 symmetry (Goldstone) modes in red, and polar modes in green. The frequencies of all the other phonons are temperature independent by construction in our approximation. A detailed description of the construction of the force-constant matrices is given in the Appendix.

We begin by comparing, in Table III, the frequencies obtained from our Landau theory approach in the zero-Kelvin limit with those calculated for the fully relaxed cell using

(a)

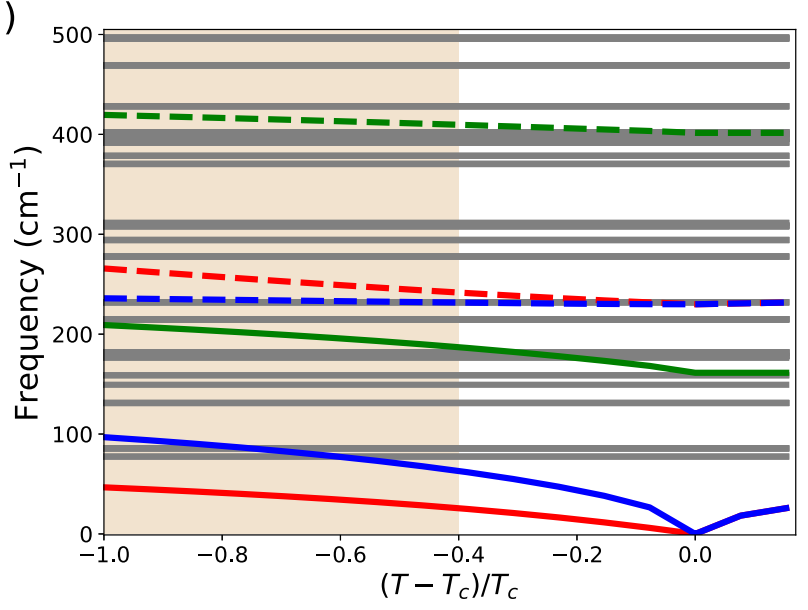

(b)

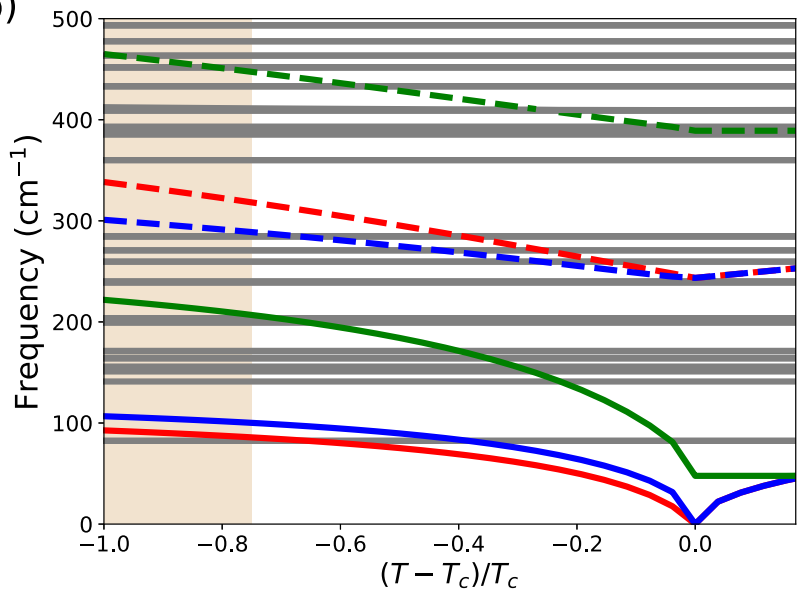

FIG. 3. Calculated evolution of phonon frequencies from the Landau theory approach for (a) ferroelectric $\mathrm{InMnO}_{3}$ and (b) $\mathrm{ErMnO}_{3}$. Modes changing due to freeze-in of the primary order parameter are marked red (Goldstone, B1) and blue (Higgs, A1), while the phonon modes related to the polar instability (A1) are marked green. Shaded areas mark the temperature range accessible by our Raman experiments. 
TABLE III. Zero-temperature frequencies of the modes with Higgs and Goldstone character in $\mathrm{ErMnO}_{3}$ and $\mathrm{InMnO}_{3}$ extrapolated from the Landau model and calculated using DFT for the relaxed low-symmetry structures. All frequencies are given in units of $\mathrm{cm}^{-1}$.

\begin{tabular}{lcccc}
\hline \hline & Higgs 1 & Goldstone 1 & Higgs 2 & Goldstone 2 \\
\hline ErMnO$_{3}$ (Landau) & 106 & 93 & 285 & 338 \\
ErMnO$_{3}$ (DFT) & 130 & 107 & 315 & 424 \\
$\mathrm{InMnO}_{3}$ (Landau) & 96 & 47 & 232 & 265 \\
$\mathrm{InMnO}_{3}$ (DFT) & 128 & 65 & 235 & 302 \\
\hline \hline
\end{tabular}

density functional theory. We find that the Landau theory frequencies underestimate the DFT values by around $30 \%$. This is mostly a result of our neglecting the weak coupling of the order parameter modes to two additional modes, as described in Ref. [37]. This additional coupling would harden the relevant phonon modes in the Landau description.

Next, we discuss the temperature dependence of the modes, beginning with the softest mode. First, we note that this mode, which softens on approaching $T_{C}$ from above, is doubly degenerate above $T_{C}$ due to the equivalence of the order-parameter directions in the high-symmetry phase. The degeneracy is lifted and the mode splits into two below the phase transition, an amplitude mode with A1 symmetry, which can be regarded as the primary Higgs mode of the structural transition (shown in blue) and a phase mode with B1 symmetry which represents the primary Goldstone mode (shown in red). The Goldstone mode in $\mathrm{InMnO}_{3}$ retains a lower frequency down to zero Kelvin than that in $\mathrm{ErMnO}_{3}$, reflecting the smaller barriers in the brim of the hat in the $\mathrm{InMnO}_{3}$ case. The Higgs modes have a similar temperature dependence and zero-Kelvin frequency in both compounds. We note, however, that the zero of $\left(\frac{T-T_{c}}{T_{c}}\right)$ corresponds to a very different temperature in the two cases $(\sim 1200 \mathrm{~K}$ for $\mathrm{ErMnO}_{3}$ and $\sim 500 \mathrm{~K}$ for $\mathrm{InMnO}_{3}$ ). We find, as expected, the occurrence of additional temperature-dependent Higgs- and Goldstone-type modes at higher frequencies, indicated with dashed lines in Fig. 3. These modes have the same symmetry as the soft modes, and their temperature dependence is a consequence of the mixing of eigenmodes caused by the transformation from the force-constant to dynamical matrices. This mixing is stronger in $\mathrm{ErMnO}_{3}$ than in $\mathrm{InMnO}_{3}$, because of the larger mass of Er, resulting in a stronger temperature dependence of the higher-frequency $\mathrm{A} 1$ and $\mathrm{B} 1$ phonons in $\mathrm{ErMnO}_{3}$.

The temperature evolutions of the phonons corresponding to the polar modes are plotted in green. These are independent of temperature above $T_{C}$, but we find that their frequencies increase below the phase transition, as the increase in magnitude of the primary order parameter stabilizes the polar mode. We see that the frequency of the polar mode increases more in $\mathrm{ErMnO}_{3}$ than $\mathrm{InMnO}_{3}$, consistent with the larger coupling $g$ in the $Q^{3} P \cos 3 \theta$ term of the Landau free energy for $\mathrm{ErMnO}_{3}$.

\section{Effect of change in lattice parameters on the phonon mode frequencies}

Finally for this section, we calculate how the change in lattice parameters with temperature affects the phonon (a)

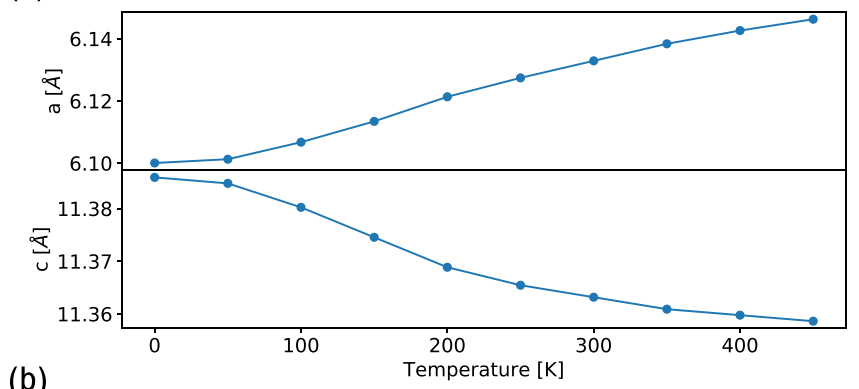

(b)

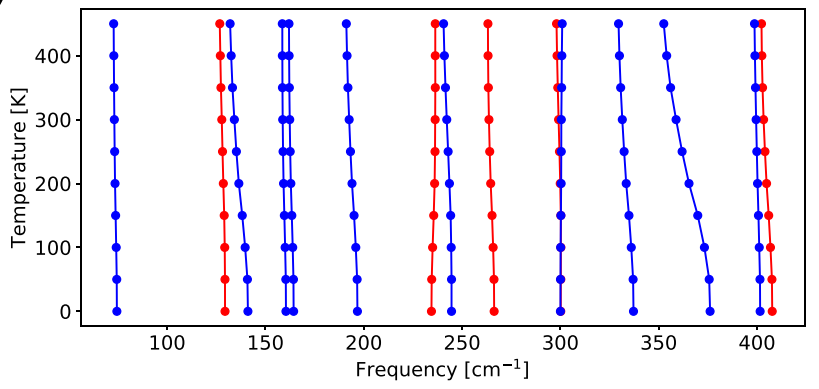

FIG. 4. (a) Lattice parameters for $\mathrm{ErMnO}_{3}$ calculated within the quasiharmonic approximation. (b) Temperature dependence of the phonon frequencies calculated for $\mathrm{ErMnO}_{3}$ within the quasiharmonic approximation. We show only the A1 (red) and E2 (blue) modes for comparison with the Raman spectroscopy measurements in the next section.

frequencies in $\mathrm{ErMnO}_{3}$, with the goal of isolating any mode softening due to thermal expansion from the mode softening due to approaching the phase transition discussed above. $\mathrm{ErMnO}_{3}$ and the other rare-earth hexagonal manganites are known experimentally to have an unusual lattice response to temperature, with the in-plane lattice parameter $a$ increasing with temperature as expected, but the out-of-plane $c$ lattice parameter decreasing with increasing temperature [57].

We begin by demonstrating that this unusual evolution can be captured within the quasiharmonic approximation, in which the total free energy as a function of the lattice parameters $F_{\text {tot }}$ is obtained by minimization of the sum of the internal energy $E(a, c)$ plus the phonon free energy $F_{\text {phonons }}(a, c)$ :

$$
F_{\text {tot }}=\min _{a, c}\left[E(a, c)+F_{\text {phonons }}(a, c)\right],
$$

where $a$ and $c$ are the in-plane and out-of-plane lattice parameters. $E(a, c)$ is obtained by relaxing all internal degrees of freedom for the $P 6_{3} \mathrm{~cm}$ structure for the set of given lattice parameters, and the phonon free energy is calculated using the partition function for harmonic phonons:

$F_{\text {phonons }}=\frac{1}{2} \sum_{q, v} \hbar \omega_{q, v}+k_{B} T \sum_{q, v} \ln \left[1-\exp \hbar \omega_{q, v} /\left(k_{B} T\right)\right]$,

where $q, v$ is the sum over the reciprocal lattice and all bands $v$.

Using this approach, we calculate the temperature dependence of the lattice parameters, which we present in Fig. 4 for $\mathrm{ErMnO}_{3}$. The excellent agreement with experiment [42] suggests that the quasiharmonic population of phonons with 
increasing temperature is the dominant contribution to the thermal evolution of the lattice parameters. We then approximate the temperature dependence of the phonon frequencies, by calculating the eigenmodes of the dynamical matrix at the $a, c$ lattice parameters for the corresponding temperature. We deliberately omit anharmonic interactions and phonon populations in this step, in order to isolate specifically the effect of the change in lattice parameters. We show our results for the Raman-active A1 and E2 phonons in Fig. 4. We find that in this limit, most modes, in particular the A1 and B1 (not shown) phonons relevant to the Higgs-Goldstone coupling, are largely temperature independent. Therefore, we can exclude that any measured temperature dependence of the Higgs and Goldstone modes is a result of the change in lattice parameters with temperature.

\section{EXPERIMENTAL RESULTS}

\section{A. Raman spectroscopy}

In Fig. 5 the Raman spectra at $10 \mathrm{~K}$ are displayed for (a) ferroelectric $P 6_{3} \mathrm{~cm} \mathrm{ErMnO}_{3}$, (b) $\mathrm{InMnO}_{3}$ in the antipolar $P \overline{3} c 1$ state, and (c) the ferroelectric $P 6_{3} \mathrm{~cm}$ variant of $\mathrm{InMnO}_{3}$, for parallel (red) and perpendicular (black) polarizations of incoming and scattered photons. Our $\mathrm{ErMnO}_{3}$ data [Fig. 5(a)] are in excellent agreement with previously published results [58], showing all the previously reported A1 and E2 Raman-active modes with the expected relative intensities and positions. The extinction of the A1 modes in the perpendicular configuration confirms the selection rules for the $P 6_{3} \mathrm{~cm}$ space group, and the narrow linewidths confirm the high quality of the $\mathrm{ErMnO}_{3}$ single crystal used in this study. For both the $P 6_{3} \mathrm{~cm}$ and $P \overline{3} c 1 \mathrm{InMnO}_{3}$ crystals we observe in Figs. 5(b) and 5(c) the extinction of the mode at $680 \mathrm{~cm}^{-1}$ and of the shoulder at $280 \mathrm{~cm}^{-1}$ for the perpendicular polarizer configuration, which indicates that these modes belong to the A1 representation. The peaks at $\sim 140,280$, and $330 \mathrm{~cm}^{-1}$ persist for perpendicular polarization and therefore have E2 symmetry. The small crystal size leads to broad peaks and difficulty in umambiguously assigning the remaining peak frequencies, although the peaks at around 450 and $600 \mathrm{~cm}^{-1}$ are likely of A1 symmetry.

In Figs. 6(a)-6(c) we show the detailed temperature dependence of the Raman spectra of all three crystals. For the sake of clarity, the curves have been shifted vertically proportional to their temperatures. By fitting the curves with Lorentzian functions, we extracted the temperature dependence of the phonon frequencies, shown in Fig. 6(d) for the $\mathrm{ErMnO}_{3}$ sample, Fig. 6(e) for the $P \overline{3} c 1 \mathrm{InMnO}_{3}$ sample, and Fig. 6(f) for the $P 6_{3} \mathrm{~cm} \mathrm{InMnO}_{3}$ sample.

We begin by analyzing the $\mathrm{ErMnO}_{3}$ spectrum, which shows all the A1 and E2 Raman-active modes reported previously in the literature [58] with the expected relative intensities and positions. Moreover, we observe a new small peak below $80 \mathrm{~cm}^{-1}$ that was not resolved in the $10-\mathrm{K}$ spectrum and increases in intensity with increasing temperature. Our calculations indicate that this is an E2 (and therefore Raman-active) mode, and its temperature dependence is likely the result of low Raman activity, which increases due to anharmonic mixing with other E2 modes as the temperature is increased.

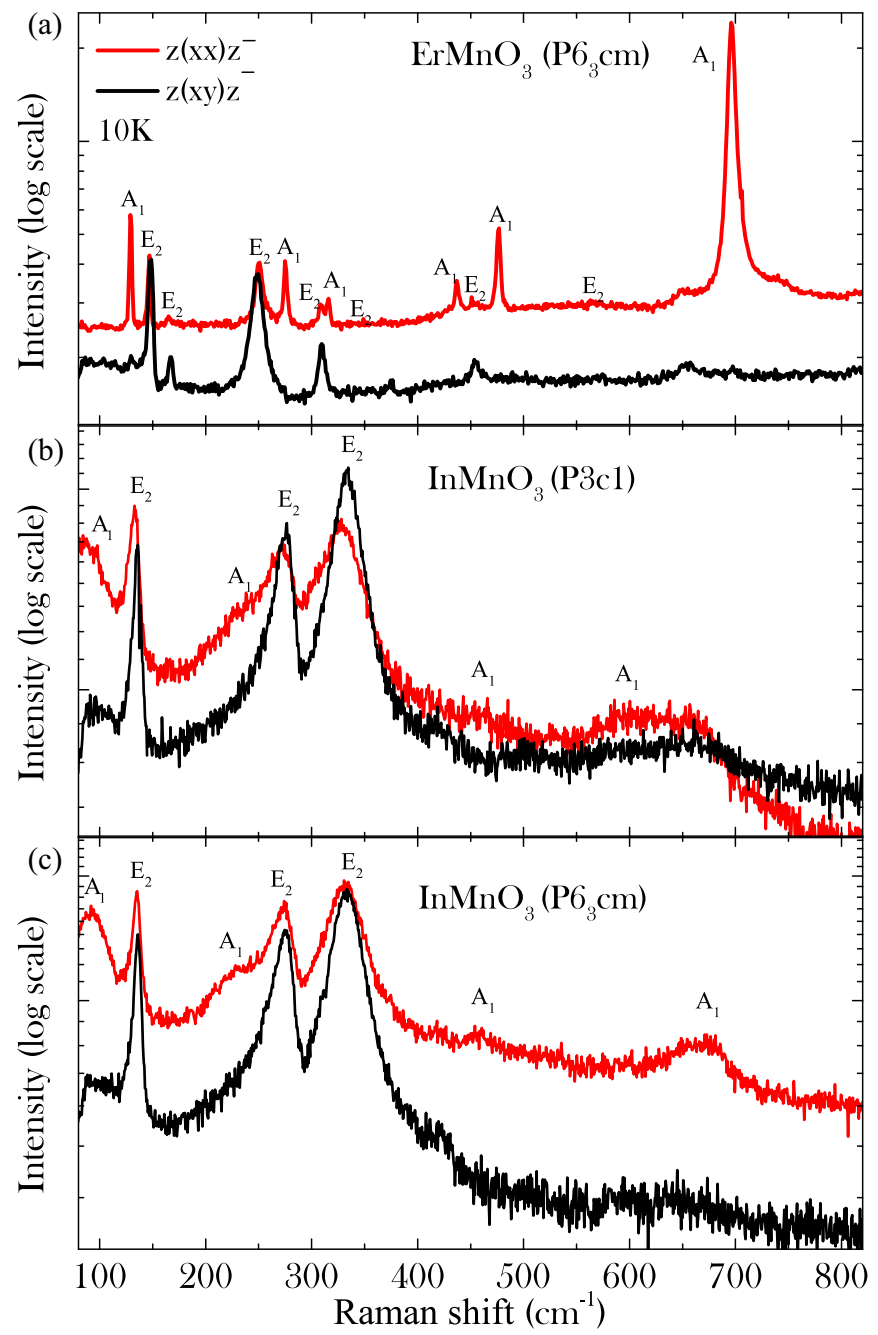

FIG. 5. Raman spectra collected at $10 \mathrm{~K}$ on single crystals of (a) $\mathrm{ErMnO}_{3}$, (b) $\mathrm{InMnO}_{3}(\mathrm{P} \overline{3} \mathrm{c} 1)$, and (c) $\mathrm{InMnO}_{3}\left(\mathrm{P}_{3} \mathrm{~cm}\right)$. The red and black lines show the intensity of Raman scattering in the parallel and perpendicular configurations, respectively. The polarization selection rule for the $\mathrm{ErMnO}_{3}$ sample ( $P 6_{3} \mathrm{~cm}$ symmetry) is clearly manifested.

We also observe a general softening of all the modes as the temperature is increased. The frequency of the lowestfrequency A1 mode, which has the strongest Higgs character, reduces by $\sim 10 \mathrm{~cm}^{-1}$ between 10 and $300 \mathrm{~K}$, with the higherenergy A1 modes reducing in frequency by a similar amount. Since the ferroelectric phase transition in $\mathrm{ErMnO}_{3}$ occurs at $\sim 1200 \mathrm{~K}$, the $300-\mathrm{K}$ limit of our experiment corresponds to $\frac{T-T_{C}}{T_{C}}=-0.75$, which we see from Fig. 3(b) corresponds to a predicted Landau theory drop in frequency of around $10 \mathrm{~cm}^{-1}$, consistent with the experiment. The high $T_{C}$ of $\mathrm{ErMnO}_{3}$ means that definitive experimental confirmation of Higgs behavior in $\mathrm{ErMnO}_{3}$ would require measurement of the phonon frequencies to higher temperature than is available in our setup. The E1 mode at $250 \mathrm{~cm}^{-1}$ shows a particularly strong broadening and redshift; we suggest that this corresponds to a shear mode which we find in our quasiharmomic 


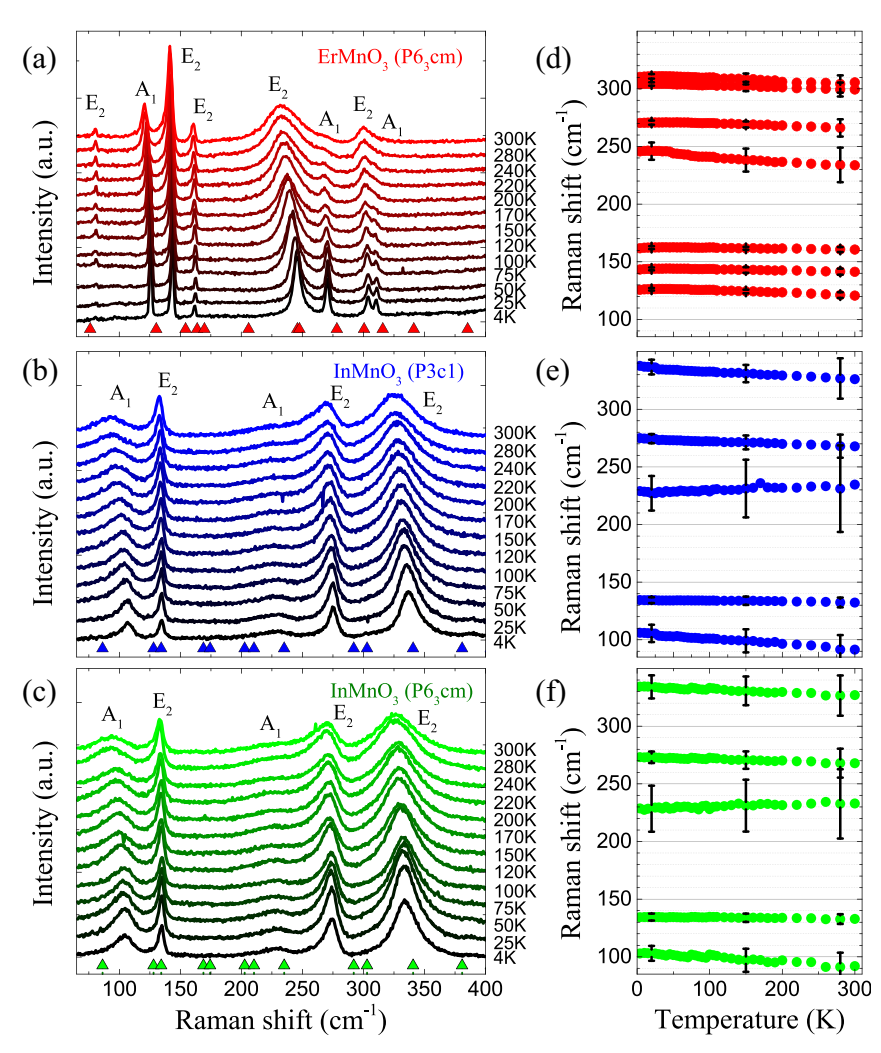

FIG. 6. Raman spectra at selected temperatures from 4 to $300 \mathrm{~K}$ for three hexagonal manganite samples: (a) $\mathrm{ErMnO}_{3}$, (b) $\mathrm{InMnO}_{3}$ $(\mathrm{P} \overline{3} \mathrm{c} 1)$, and $(\mathrm{c}) \mathrm{InMnO}_{3}\left(\mathrm{P}_{3} \mathrm{~cm}\right)$. The colored triangles mark calculated values from our DFT calculations. (d)-(f) Temperature dependence of the mode frequencies for the three samples: (d) $\mathrm{ErMnO}_{3}$, (e) $\mathrm{InMnO}_{3}(\mathrm{P} \overline{3} \mathrm{c} 1)$, and (f) $\mathrm{InMnO}_{3}\left(\mathrm{P}_{3} \mathrm{~cm}\right)$. The black vertical bars indicate the peak widths.

calculations to be particularly sensitive to the change in lattice parameters.

Next, we analyze the $\mathrm{InMnO}_{3}$ spectra. Our first observation is that, despite their different ground-state crystal structures, the Raman spectra of the two $\mathrm{InMnO}_{3}$ crystals are almost identical, confirming the similarity in the shapes of their Mexican hat potentials. In both $\mathrm{InMnO}_{3}$ cases, the main Higgs excitation associated with the lowest-frequency mode is lower in frequency and softens more rapidly with increasing temperature than in $\mathrm{ErMnO}_{3}$, consistent with the lower Curie temperature of $\sim 500 \mathrm{~K}$. Once again we find a good agreement with the Landau theory prediction, with the calculated drop in frequency between 0 and $300 \mathrm{~K}$ (corresponding to $\frac{T-T_{C}}{T_{C}}=$ -0.4 ) of around $30 \mathrm{~cm}^{-1}$ compared with the measured value of $\sim 20 \mathrm{~cm}^{-1}$. Interestingly, for $\mathrm{InMnO}_{3}$ the E2 modes show a much weaker temperature dependence than in $\mathrm{ErMnO}_{3}$, with the mode at $135 \mathrm{~cm}^{-1}$ largely temperature independent and the mode at $225 \mathrm{~cm}^{-1}$ even hardening upon increasing the temperature. We attribute this behavior to the In-O covalency, which is known to lead to a larger $c$ lattice parameter for $\mathrm{InMnO}_{3}$ compared to other members of the hexagonal manganite series [54], and likely also causes markedly different changes in lattice parameters with thermal expansion [59].

\section{SUMMARY}

In summary, we have analyzed the role of phonons as Higgs and Goldstone modes at the structural phase transitions in crystalline materials, focusing particularly on the case of the hexagonal manganites. We showed that, in materials containing atoms of more than one mass, the static Higgs and Goldstone modes only map uniquely onto single-phonon modes at $T_{C}$, where both the Higgs and Goldstone frequencies are zero. Below $T_{C}$, the different masses of the ions cause a softening of several phonon modes with the same symmetry as the static soft-mode distortion. Nevertheless, in both $\mathrm{ErMnO}_{3}$ and $\mathrm{InMnO}_{3}$, our Landau theory analysis identified one primary A1 phonon corresponding to the Higgs mode, and one main B1 phonon corresponding to the Goldstone-like mode. We then used Landau theory with parameters from density functional theory to calculate the temperature evolution of these modes, noting that the formalism is strictly only valid for displacive phase transitions, in which the structural distortion is zero in the high-temperature phase.

Using Raman spectroscopy, we showed that the lowest A1 modes in both $\mathrm{ErMnO}_{3}$ and $\mathrm{InMnO}_{3}$ indeed have a redshift in the frequency on warming. For $\mathrm{InMnO}_{3}$, in which the temperature range measured is substantial with respect to the Curie temperature, the magnitude of the shift is also substantial, and similar to that predicted by the Landau theory. The good match between the temperature evolution of the Higgs mode in our Raman measurements and in our calculations using Landau and density functional theories suggests that the phase transition in $\mathrm{InMnO}_{3}$ is well described within a standard displacive picture. A definitive measurement of the temperature dependence of the Higgs mode in $\mathrm{ErMnO}_{3}$ will require Raman or inelastic neutron scattering measurements to higher temperatures. To motivate such measurements, we suggest that $\mathrm{ErMnO}_{3}$ might show intriguing deviations from the temperature evolution that we calculated within Landau theory since it will likely have at least partial order-disorder character, in which the high-temperature structure is locally distorted, but the distortion vanishes on average. Such orderdisorder behavior was recently identified in the related material $\mathrm{YMnO}_{3}$ [42], where inelastic neutron scattering indicated only limited phonon softening [60-62]. We suggest that $\mathrm{ErMnO}_{3}$ is a good candidate for detection of the predicted emergence of a central peak at the transition (see for example [63]), which has so far proved elusive.

\section{ACKNOWLEDGMENTS}

We thank M. Bieringer, Department of Chemistry, University of Manitoba, for providing the $\mathrm{InMnO}_{3}$ single crystals and K. Kohn, Waseda University, for providing the $\mathrm{ErMnO}_{3}$ single crystals. We also thank A. Cano and A. Mozzafari for helpful discussions and M. Miller for graphical assistance. Computing resources were provided by the Euler cluster at ETHZ and CSCS under project IDs eth3 and s889. This work was supported by the Körber Foundation, by the Swiss National Science Foundation through the National Center of Competence in Research (NCCR) MARVEL, by the ETH Zurich, and has received funding from the European Research Council (ERC) under the European Union's Horizon 2020 research 
and innovation programme Grant Agreement No. 810451. S.M.G. was supported by the Quantum Information Science Enabled Discovery (QuantISED) for High Energy Physics (Grant No. KA2401032) at LBNL. Work at the Molecular Foundry (S.M.G) was supported by the Office of Science, Office of Basic Energy Sciences, of the U. S. Department of Energy under Contract No. DE-AC02-05CH11231.

\section{APPENDIX A: TECHNICAL DETAILS OF THE COMBINED DFT/LANDAU THEORY TREATMENT}

\section{Perfect Mexican hat}

In this Appendix we discuss how we combine Landau theory with density functional theory calculations to obtain the phonon spectrum as a function of the atmonic displacement. We start by calculating the force-constant matrix of the high-symmetry phase $\left(\Phi_{i j}\right)$, which is the second derivative of the Kohn-Sham total energy $E_{\mathrm{KS}}$ with respect to atomic displacements $u_{i}, u_{j}\left(\Phi_{i j}=\frac{\partial E_{K S}}{\partial u_{i} \partial u_{j}}\right)$ in the limit of small $u_{i}$. As this is a harmonic treatment, the generalized susceptibility $\chi_{i i}^{-1}=\frac{\partial^{2} E_{K S}}{\partial \phi_{i}^{2}}$ for an eigenmode $\phi_{i}$ is the corresponding eigenvalue of the $\Phi_{i j}$ matrix. For a system with $N$ atoms in the unit cell, $\Phi_{i j}$ is a $3 N \times 3 N$ matrix, and we label the eigenvalues $\alpha_{1}, \ldots, \alpha_{3 N}$. In the structural ground state, all eigenvalues are positive. Negative eigenvalues occur when atomic displacements do not induce restoring forces and correspond to energy lowering structural distortions.

Since structural phase transitions are determined by the eigenvectors with negative eigenvalues, next we treat their anharmonicity, while assuming that they have no interaction with the stable modes. We assume without loss of generality that we have two negative, degenerate eigenvalues, $\alpha_{1}, \alpha_{2}$ say, and we take $\alpha_{3}, \ldots \alpha_{3 N}$ to be constant with temperature. In order to incorporate the anharmonicity of these modes in our Landau potential, we perform a series of calculations of the Kohn-Sham energy, for several amplitudes of each mode eigenvector superimposed onto the high-symmetry structure. We then fit a Landau polynomial to the resulting energy landscape. In the case of of a perfect Mexican hat potential, the form of the energy landscape is

$$
E_{\mathrm{KS}}\left(\phi_{1}, \phi_{2}\right)=\frac{a}{2}\left(\phi_{1}^{2}+\phi_{2}^{2}\right)+\frac{b}{4}\left(\phi_{1}^{2}+\phi_{2}^{2}\right)^{2},
$$

where one can extract the parameters $a$ and $b$ by fitting to the calculated $E_{\mathrm{Ks}}$. We then take into account the temperature by allowing the $a$ coefficients to be temperature dependent with the usual Landau form $a(T)=a_{0} \frac{T-T_{C}}{T_{C}}$, giving $a(T)<0$ below $T_{C}$ and $a(T)>T_{C}$ above $T_{C}$. Above $T_{C}$, the expectation values of $\phi_{1}$ and $\phi_{2}$ are equivalently zero, and the inverse generalized susceptibility matrix has the form

$$
\chi_{11}^{-1}=\left.\frac{\partial^{2} F}{\partial \phi_{1}^{2}}\right|_{\phi_{1}=0, \phi_{2}=0}=\chi_{22}^{-1}=\left.\frac{\partial^{2} F}{\partial \phi_{2}^{2}}\right|_{\phi_{1}=0, \phi_{2}=0}=a(T) .
$$

Making use of our assumption that $\alpha_{3}, \ldots, \alpha_{3 N}$ are temperature independent, and the entire temperature dependence of the free energy is contained in $\alpha_{1}$ and $\alpha_{2}$, the susceptibility matrix above $T_{C}$ is then

$$
\chi^{-1}(T)=\left[\begin{array}{ccccc}
a(T) & 0 & 0 & \ldots & 0 \\
0 & a(T) & 0 & \ldots & 0 \\
0 & 0 & \alpha_{3} & \ldots & 0 \\
\vdots & \vdots & \vdots & \ddots & \vdots \\
0 & 0 & 0 & \ldots & \alpha_{3 N}
\end{array}\right] .
$$

Below $T_{C}, \phi_{1}$ and $\phi_{2}$ correspond to the Goldstone and Higgs modes. The Goldstone mode has an eigenvalue of zero for all temperatures below the phase transition. The Higgs mode, in contrast, softens with increasing temperature, so that its eigenvalue goes to zero at the phase transition. Formally, we obtain the solutions below $T_{C}$ by minimizing the free energy $F$ [Eq. (4)] with respect to $\phi_{1}$ and $\phi_{2}$ to extract the expectation value of the order parameter. This yields two solutions, the trivial vacuum solution with $\left\langle\phi_{1}\right\rangle=\left\langle\phi_{2}\right\rangle=$ 0 for $-a(T) / b>0$, and a nontrivial solution describing a degenerate circle of vacua $\phi_{1}^{2}+\phi_{2}^{2}=\langle\phi\rangle=-a(T) / b$, which is the Mexican hat potential. Because of the $\mathrm{U}(1)$ symmetry we can choose $\left\langle\phi_{1}\right\rangle=0$ and $\left\langle\phi_{2}\right\rangle=\langle\phi\rangle$ without loss of generality, and expand around the low-symmetry vacuum ground state to obtain the excitation modes. This gives the massless Goldstone mode, corresponding to distortions along the $\phi_{1}$ coordinate around the brim of the hat $\delta \phi_{1}$, with

$$
\phi_{1}=\delta \phi_{1},
$$

and the massive Higgs mode, corresponding to distortions along the perpendicular $\phi_{2}$ coordinate $\delta \phi_{2}$, with

$$
\phi_{2}=\langle\phi\rangle+\delta \phi_{2}=\delta \phi_{2}-a(T) / b .
$$

The modes satisfy

$$
\left\langle\delta \phi_{1}\right\rangle=\left\langle\delta \phi_{2}\right\rangle=0 .
$$

The frequencies of the Goldstone and Higgs modes are

$$
\begin{gathered}
\chi_{11}^{-1}(T)=\left.\frac{\partial^{2} F}{\partial \phi_{2}^{2}}\right|_{\phi_{1}=0, \phi_{2}=\langle\phi\rangle}=0, \\
\chi_{22}^{-1}(T)=\left.\frac{\partial^{2} F}{\partial \phi_{1}^{2}}\right|_{\phi_{1}=0, \phi_{2}=\langle\phi\rangle}=a(T)+3 b\langle\phi\rangle^{2}=-2 a(T)
\end{gathered}
$$

and the whole susceptibility matrix below $T_{C}$ is

$$
\chi^{-1}(T)=\left[\begin{array}{ccccc}
0 & 0 & 0 & \ldots & 0 \\
0 & -2 a(T) & 0 & \ldots & 0 \\
0 & 0 & \alpha_{3} & \ldots & 0 \\
\vdots & \vdots & \vdots & \ddots & \vdots \\
0 & 0 & 0 & \ldots & \alpha_{3 N}
\end{array}\right] .
$$

Finally, we remark that to obtain the phonon frequencies, that is the eigenvalues of the dynamical matrix, as opposed to the eigenvalues of the force-constant matrix that we have analyzed here, we first have to transform the inverse susceptibility back to the force-constant 
matrix using $\Phi=M^{-1} \chi^{-1} M$ where $M$ is the transformation matrix from the diagonalization. The phonon frequencies are then obtained by diagonalizing the dynamical matrix $D_{i j}$ :

$$
D_{i j}=\frac{\Phi_{i j}}{\sqrt{M_{i} M_{j}}} .
$$

\section{2. $\mathrm{h}-\mathrm{RMnO}_{3}$}

The case of $\mathrm{h}-R \mathrm{MnO}_{3}$ is similar to the simple case discussed in the previous section in that the structural transition in the hexagonal manganites is described by a two-dimensional (2D) primary order parameter parametrized by $(Q, \Phi)$. In addition, we now have to take into account the improper coupling of the primary order parameter to the secondary order parameter $P$. The Landau Free energy is then given by

$$
\begin{aligned}
F= & \frac{a(T)}{2} Q^{2}+\frac{b}{4} Q^{4}+\frac{1}{6}\left(c+c^{\prime} \cos 6 \theta\right) Q^{6} \\
& -g Q^{3} P \cos 3 \theta+\frac{g^{\prime}}{2} Q^{2} P^{2}+\frac{a_{p}}{2} P^{2} .
\end{aligned}
$$

Above $T_{C}$, the inverse susceptibility matrix has the form

$$
\chi^{-1}(T)=\left[\begin{array}{ccccccc}
a(T) & 0 & 0 & 0 & 0 & \ldots & 0 \\
0 & a(T) & 0 & 0 & 0 & \ldots & 0 \\
0 & 0 & a_{P} & 0 & 0 & \ldots & 0 \\
0 & 0 & 0 & \alpha_{4} & 0 & \ldots & 0 \\
\vdots & \vdots & \vdots & \vdots & \vdots & \ddots & \vdots \\
0 & 0 & 0 & 0 & 0 & \ldots & \alpha_{3 N}
\end{array}\right]
$$

Calculating perturbations of the order parameter below $T_{C}$ we obtain the mode inverse susceptibilities:

$$
\begin{gathered}
\chi_{\delta q}^{-1}(T)=\left.\frac{\partial^{2} F}{\partial Q^{2}}\right|_{Q=\tilde{Q}, \theta=0, P=\tilde{P}}=a(T)+3 b \tilde{Q}^{2}+5 b \tilde{Q}^{4}\left(c+c^{\prime}\right)-6 g \tilde{Q} \tilde{P}+g^{\prime} \tilde{P}^{2}, \\
\chi_{\delta \theta}^{-1}(T)=\left.\frac{1}{Q^{2}} \frac{\partial^{2} F}{\partial \theta^{2}}\right|_{Q=\tilde{Q}, \theta=0, P=\tilde{P}}=-6 \tilde{Q}^{4}+9 g \tilde{Q} \tilde{P}, \\
\chi_{\delta p}^{-1}(T)=\left.\frac{\partial^{2} F}{\partial P^{2}}\right|_{Q=\tilde{Q}, \theta=0, P=\tilde{P}}=g^{\prime} \tilde{Q}^{2}+a_{p}
\end{gathered}
$$

and thus the matrix

$$
\chi^{-1}(T)=\left[\begin{array}{ccccccc}
\chi_{\delta q}^{-1}(T) & 0 & 0 & 0 & 0 & \ldots & 0 \\
0 & \chi_{\delta \theta}^{-1}(T) & 0 & 0 & 0 & \ldots & 0 \\
0 & 0 & \chi_{\delta p}^{-1}(T) & 0 & 0 & \ldots & 0 \\
0 & 0 & 0 & \alpha_{4} & 0 & \ldots & 0 \\
\vdots & \vdots & \vdots & \vdots & \vdots & \ddots & \vdots \\
0 & 0 & 0 & 0 & 0 & \ldots & \alpha_{3 N}
\end{array}\right]
$$

The phonon frequencies are then again found by transforming back to the force-constant matrix, normalizing by the atomic masses and diagonalizing the resulting dynamical matrix. 


\section{APPENDIX B: LIST OF CALCULATED PHONON FREQUENCIES}

TABLE IV. Calculated zero-Kelvin DFT phonon frequencies for $\mathrm{InMnO}_{3}$ (left) and $\mathrm{ErMnO}_{3}$ (right).

\begin{tabular}{|c|c|c|c|}
\hline Frequency $\left(\mathrm{cm}^{-1}\right)$ & Irrep & Frequency $\left(\mathrm{cm}^{-1}\right)$ & Irrep \\
\hline 64.8 & B1 & 76.3 & E2 \\
\hline 86.3 & E2 & 84.8 & A2 \\
\hline 114.4 & B2 & 107.3 & B1 \\
\hline 128.0 & A1 & 130.3 & A1 \\
\hline 134.3 & E2 & 154.3 & E2 \\
\hline 168.9 & E2 & 162.1 & E1 \\
\hline 174.3 & E2 & 163.9 & E2 \\
\hline 176.0 & $\mathrm{E} 1$ & 169.8 & E2 \\
\hline 178.3 & B2 & 171.0 & E1 \\
\hline 189.2 & E2 & 206.0 & E2 \\
\hline 210.3 & E2 & 256.2 & E1 \\
\hline 216.1 & E1 & 263.2 & A2 \\
\hline 230.7 & $\mathrm{~A} 2$ & 270.2 & B1 \\
\hline 235.1 & A1 & 271.5 & B2 \\
\hline 255.8 & B2 & 278.3 & A1 \\
\hline 272.1 & E1 & 279.1 & B1 \\
\hline 289.1 & A 2 & 293.4 & E1 \\
\hline 292.0 & E2 & 300.5 & E2 \\
\hline 303.0 & A1 & 315.2 & A2 \\
\hline 302.9 & B1 & 315.9 & A1 \\
\hline 307.8 & B2 & 341.3 & E2 \\
\hline 340.7 & E2 & 347.4 & B2 \\
\hline 408.9 & E1 & 424.0 & B1 \\
\hline 410.2 & E2 & 426.2 & A1 \\
\hline 429.6 & A2 & 433.1 & E1 \\
\hline 429.9 & B1 & 451.2 & E2 \\
\hline 445.7 & B2 & 454.5 & E2 \\
\hline 446.9 & A1 & 456.5 & E1 \\
\hline 470.8 & A1 & 458.2 & B2 \\
\hline 494.0 & B2 & 463.7 & A1 \\
\hline 494.8 & A2 & 494.7 & E1 \\
\hline 495.6 & B1 & 495.7 & E2 \\
\hline 501.7 & E2 & 505.0 & A1 \\
\hline 509.7 & E1 & 521.2 & B1 \\
\hline 527.5 & E1 & 526.4 & A2 \\
\hline 533.2 & E2 & 535.9 & B2 \\
\hline 573.7 & A1 & 603.1 & A1 \\
\hline 609.3 & $\mathrm{E} 1$ & 632.0 & E2 \\
\hline 611.6 & E2 & 632.3 & E1 \\
\hline 614.8 & E2 & 637.4 & E2 \\
\hline 618.9 & $\mathrm{E} 1$ & 637.9 & E1 \\
\hline 645.4 & B2 & 686.2 & B2 \\
\hline 657.0 & A1 & 688.9 & A1 \\
\hline 736.5 & B2 & 771.5 & B2 \\
\hline
\end{tabular}


[1] K. Baumann, C. Guerlin, F. Brennecke, and T. Esslinger, Nature (London) 464, 1301 (2010).

[2] L. Sadler, J. Higbie, S. Leslie, M. Vengalattore, and D. StamperKurn, Nature (London) 443, 312 (2006).

[3] M. Tinkham, Introduction to Superconductivity (Dover, New York, 1996).

[4] CMS Collaboration, Phys. Lett. B 716, 30 (2012).

[5] F. Englert and R. Brout, Phys. Rev. Lett. 13, 321 (1964).

[6] P. W. Higgs, Phys. Rev. Lett. 13, 508 (1964).

[7] G. S. Guralnik, C. R. Hagen, and T. W. B. Kibble, Phys. Rev. Lett. 13, 585 (1964).

[8] ATLAS Collaboration, Phys. Lett. B 716, 1 (2012).

[9] L. D. Landau and E. M. Lifshitz, Statistical Physics (Pergamon, London, 1958).

[10] L. P. Kadanoff, W. Götze, D. Hamblen, R. Hecht, E. A. Lewis, J. Swift, D. Aspnes, and J. Kane, Rev. Mod. Phys. 39, 395 (1967).

[11] M. E. Peskin and D. V. Schroeder, An Introduction to Quantum Field Theory (Avalon, New York, 1995).

[12] J. Goldstone, Il Nuovo Cimento 19, 154 (1961).

[13] J. Goldstone, A. Salam, and S. Weinberg, Phys. Rev. 127, 965 (1962).

[14] P. Böni, M. Hennion, and J. L. Martínez, Phys. Rev. B 52, 10142 (1995).

[15] C. Rüegg, N. Cavadini, A. Furrer, H.-U. Güdel, K. Krämer, H. Mutka, A. Wildes, K. Habicht, and P. Vorderwisch, Nature (London) 423, 62 (2003).

[16] M. Matsumoto, B. Normand, T. M. Rice, and M. Sigrist, Phys. Rev. Lett. 89, 077203 (2002).

[17] C. A. Kendziora, I. A. Sergienko, R. Jin, J. He, V. Keppens, B. C. Sales, and D. Mandrus, Phys. Rev. Lett. 95, 125503 (2005).

[18] S. M. Nakhmanson and I. Naumov, Phys. Rev. Lett. 104, 097601 (2010).

[19] N. N. Kovaleva, O. E. Kusmartseva, K. I. Kugel, A. A. Maksimov, D. Nuzhnyy, A. M. Balbashov, E. I. Demikhov, A. Dejneka, V. A. Trepakov, F. V. Kusmartsev, and A. M. Stoneham, J. Phys.: Condens. Matter 25, 155602 (2013).

[20] G. E. Volovik, Exotic Properties of Superfluid 3He, Vol. 1 (World Scientific, Singapore, 1992).

[21] P. W. Anderson, Phys. Rev. 110, 827 (1958).

[22] P. W. Anderson, Phys. Rev. 112, 1900 (1958).

[23] D. Podolsky, A. Auerbach, and D. P. Arovas, Phys. Rev. B 84, 174522 (2011).

[24] P. B. Littlewood and C. M. Varma, Phys. Rev. Lett. 47, 811 (1981).

[25] R. Sooryakumar and M. V. Klein, Phys. Rev. Lett. 45, 660 (1980).

[26] C. Rüegg, B. Normand, M. Matsumoto, A. Furrer, D. F. McMorrow, K. W. Krämer, H.-U. Güdel, S. N. Gvasaliya, H. Mutka, and M. Boehm, Phys. Rev. Lett 100, 205701 (2008).

[27] M. Endres, T. Fukuhara, D. Pekker, M. Cheneau, P. Schauss, C. Gross, E. Demler, S. Kuhr, and I. Bloch, Nature (London) 487, 454 (2012).

[28] L. Pollet and N. Prokof'ev, Phys. Rev. Lett. 109, 010401 (2012).

[29] A. Marthinsen, S. M. Griffin, M. Moreau, T. Grande, T. Tybell, and S. M. Selbach, Phys. Rev. Mater. 2, 014404 (2018).
[30] J. Léonard, A. Morales, P. Zupancic, T. Donner, and T. Esslinger, Science 358, 1415 (2017).

[31] D. Pekker and C. Varma, Annu. Rev. Condens. Matter Phys. 6, 269 (2015).

[32] M. Vallone, Phys. Status Solidi B 257, 1900443 (2020).

[33] S. Artyukhin, K. T. Delaney, N. A. Spaldin, and M. Mostovoy, Nat. Mater. 13, 42 (2014).

[34] S. M. Griffin, M. Lilienblum, K. T. Delaney, Y. Kumagai, M. Fiebig, and N. A. Spaldin, Phys. Rev. X 2, 041022 (2012).

[35] P. C. Hohenberg and A. P. Krekhov, Phys. Rep. 572, 1 (2015).

[36] M. T. Dove, Introduction to Lattice Dynamics, Vol. 4 (Cambridge University Press, Cambridge, 1993).

[37] C. J. Fennie and K. M. Rabe, Phys. Rev. B 72, 100103(R) (2005).

[38] Q. N. Meier, M. Lilienblum, S. M. Griffin, K. Conder, E. Pomjakushina, Z. Yan, E. Bourret, D. Meier, F. Lichtenberg, E. K. H. Salje, N. A. Spaldin, M. Fiebig, and A. Cano, Phys. Rev. X 7, 041014 (2017).

[39] S. C. Abrahams, Acta Crystallogr., Sect. B Struct. Sci 57, 485 (2001).

[40] J. V. José, L. P. Kadanoff, S. Kirkpatrick, and D. R. Nelson, Phys. Rev. B 16, 1217 (1977).

[41] D. J. Amit and L. Peliti, Ann. Phys. 140, 207 (1982).

[42] S. H. Skjærvø, Q. N. Meier, M. Feygenson, N. A. Spaldin, S. J. L. Billinge, E. S. Bozin, and S. M. Selbach, Phys. Rev. X 9, 031001 (2019).

[43] F.-T. Huang, X. Wang, S. M. Griffin, Y. Kumagai, O. Gindele, M.-W. Chu, Y. Horibe, N. A. Spaldin, and S.-W. Cheong, Phys. Rev. Lett. 113, 267602 (2014).

[44] B. M. Wanklyn, J. Mater. Sci. 7, 813 (1972).

[45] H. Sugie, N. Iwata, and K. Kohn, J. Phys. Soc. Jpn. 71, 1558 (2002).

[46] J. E. Greedan, M. Bieringer, J. F. Britten, D. M. Giaquinta, and H.-C. zur Loye, J. Solid State Chem. 116, 118 (1995).

[47] F.-T. Huang, X. Wang, Y. S. Oh, K. Kurushima, S. Mori, Y. Horibe, and S.-W. Cheong, Phys. Rev. B 87, 184109 (2013).

[48] S. M. Griffin, M. Reidulff, S. M. Selbach, and N. A. Spaldin, Chem. Mater. 29, 2425 (2017).

[49] X. Gonze, J. M. Beuken, R. Caracas, F. Detraux, M. Fuchs, G. M. Rignanese, L. Sindic, M. Verstraete, G. Zerah, F. Jollet, M. Torrent, A. Roy, M. Mikami, P. Ghosez, J. Y. Raty, and D. C. Allan, Comput. Mater. Sci. 25, 478 (2002).

[50] X. Gonze, B. Amadon, P. M. Anglade, J. M. Beuken, F. Bottin, P. Boulanger, F. Bruneval, D. Caliste, R. Caracas, M. Côté, T. Deutsch, L. Genovese, P. Ghosez, M. Giantomassi, S. Goedecker, D. R. Hamann, P. Hermet, F. Jollet, G. Jomard, S. Leroux et al., Comput. Phys. Commun. 180, 2582 (2009).

[51] B. Amadon, F. Jollet, and M. Torrent, Phys. Rev. B 77, 155104 (2008).

[52] M. Torrent, F. Jollet, F. Bottin, G. Zérah, and X. Gonze, Comput. Mater. Sci. 42, 337 (2008).

[53] F. Jollet, M. Torrent, and N. Holzwarth, Comput. Phys. Commun. 185, 1246 (2014).

[54] Y. Kumagai, A. A. Belik, M. Lilienblum, N. Leo, M. Fiebig, and N. A. Spaldin, Phys. Rev. B 85, 174422 (2012).

[55] A. Togo and I. Tanaka, Scr. Mater. 108, 1 (2015). 
[56] D. R. Småbråten, Q. N. Meier, S. H. Skjærvø, K. Inzani, D. Meier, and S. M. Selbach, Phys. Rev. Mater. 2, 114405 (2018).

[57] A. S. Gibbs, K. S. Knight, and P. Lightfoot, Phys. Rev. B 83, 094111 (2011).

[58] J. Vermette, S. Jandl, and M. M. Gospodinov, J. Phys.: Condens. Matter 20, 425219 (2008).

[59] M. F. Bekheet, I. Svoboda, N. Liu, L. Bayarjargal, E. Irran, C. Dietz, R. W. Stark, R. Riedel, and A. Gurlo, J. Solid State Chem. 241, 54 (2016).
[60] M. K. Gupta, R. Mittal, M. Zbiri, N. Sharma, S. Rols, H. Schober, and S. L. Chaplot, J. Mater. Chem. C 3, 11717 (2015).

[61] D. Bansal, J. L. Niedziela, R. Sinclair, V. O. Garlea, D. L. Abernathy, S. Chi, Y. Ren, H. Zhou, and O. Delaire, Nat. Commun. 9, 1 (2018).

[62] H. Bouyanfif, A. M. Salah, M. Zaghrioui, and M. El Marssi, Phys. Rev. B 91, 224104 (2015).

[63] S. M. Shapiro, J. D. Axe, G. Shirane, and T. Riste, Phys. Rev. B 6, 4332 (1972). 